\title{
Joint decisions for Cold Chain Cross-border E-commerce with Overseas Warehouse Logistics Outsourcing
}

\author{
Ying-Mei Jiang ${ }^{1 *}$, Jin-Jin Mou ${ }^{1}$ \\ ${ }^{1}$ School of Management, Shandong Vocational University of Foreign Affairs, Weihai 264504, China
}

\begin{abstract}
Considering a two-echelon supply chain consisting of one overseas warehouse logistics provider and one cross-border e-commerce retailer with cold chain products over a finite time horizon, this paper studies the optimal decision-making of logistics pricing, freshness-keeping effort level and cold chain product pricing under the Stackelberg game and the cooperative game, and makes a comparative analysis of the two models. Through two-person cooperative game of transferable payment, it is proved that (1)both the total supply chain profit and individual profit of the two parties are greater than the Stackelberg game;(2) the freshness level is higher than that of the Stackelberg game;(3) the logistics price is lower than that of the Stackelberg game;(4) e-commerce transaction scale (i.e. logistics demand scale) is higher than that of the Stackelberg game; and (5) the retail price of the cold chain product is lower than the Stackelberg game. Numerical results bear out the validity of the theoretical models.
\end{abstract}

\section{Introduction}

In recent years, with the rapid development of Internet technology, cross-border e-commerce has achieved blowout growth, and has become an important way of traditional trade transformation. According to statistics, China's cross-border e-commerce transaction scale increased from 1.3 trillion Yuan in 2010 to 10.5 trillion Yuan in 2019. Of which, with the improvement of living standards, more and more consumers buy products, such as frozen fish/meat or fresh fruits from cross-border ecommerce. Cold chain products are prone to deterioration in the process of storage, transportation and other logistics links. Thus, the goods should be preserved well to guarantee the quality of the product. Thus, the development of cross-border e-commerce necessarily requires the rapid development of cross-border logistics. In detail, cross-border e-commerce logistics mode is classified into postal packet, international express service, special line logistics, overseas warehouse, etc. Low prices and wide coverage of postal packet have made it a widely used delivery mode for most cross-border e-commerce retailer, but its high loss rate and long cycle time hinders its further development greatly. To settle the drawbacks, overseas warehouse logistics integrator emerges as the times requires.

Overseas warehouse logistics is a one-stop control and management service for warehousing, sorting, packaging and delivery of goods provided by the seller of the network, foreign trade trading platform and logistics service providers independently or jointly (Sun et al., 2017). It can improve overseas customer confidence, shorten the delivery cycle, speed up, and reduce cross-border defect transaction rate(Ramanathan, 2010).

Under the environment of outsourcing the overseas warehouse logistics to a public third party, cross-border ecommerce supply chain has raised the concern for cross border e-commerce retailers to seek high profit and good logistics service/cold chain freshness level. The focus on cold chain overseas warehouse logistics operations can increase efficiency in order delivery and guarantee the freshness level by considering cross-border e-commerce requirements.

This research will make in-depth discussions on logistics price, the freshness-keeping effort level and the cooperative performance of supply chain to realize a winwin relationship between cold chain overseas warehouses logistics provider and cross-border e-commerce retailers.

The remainder of the paper is organized as follows. Section 2 presents the problem description. Section 3 constructs and compares two mathematical models for the Stackelberg game and cooperative game considered. Section 4 present numerical example analysis. Conclusions are presented in the final section.

\section{Problem description and assumptions}

This model intends to establish a two-echelon supply chain consisting of an overseas warehouse logistics provider and a cross-border e-commerce retailer with cold chain products over a finite time horizon. In this ecommerce supply chain system, the cross-border ecommerce retailer purchases cold chain products from suppliers with wholesale price $w$, and sells them to final consumers with price $P$.The overseas warehouse logistics provider is responsible for the collection of goods from suppliers, and transportation, warehousing \& storage and

"Corresponding author: jiangyingmei@yeah.net 
distribution of goods to consumers. During all the logistics process, the logistics provider provides seamless freshness-keeping effort for customers to guarantee the quality of the cold chain product. The logistics price is expressed as $L$, and logistics cost as $m$. Of which, $P>w+L>w+m$. The market demand rate generally increases with service level or freshness level and decreases with sales price (Lu, Liu,2013; Xiao, Xu, 2013; Zhang, et al, 2016; Yu, Xiao, 2017; Song et al.,2018; Li et al., 2019). In this paper, the market demand rate is depicted as $D(P, e)=A-a P+b e$, where $A$ is the inherent demand for cold chain products, $a$ is the price $(P)$ coefficient of market demand, $b$ is the sensitive factor of freshness level $(e)$. When the freshness level is nondifference, the market demand for cold chain product is also positive, that is, $A-a P>0$. The freshness-keeping effort cost of $c(e)$ is a strictly increasing differentiable function of $e$. Hence, the freshness-keeping effort cost is depicted as $c(e)=\frac{1}{2} k e^{2}$ (Lu, Liu,2013; Choi et al., 2013; Jørgensen and Zaccour,2014; Zhang et al., 2016; Yu and Xiao, 2017). Assuming that $a k>b^{2}$, and the logistics provider and cross-border e-commerce retailer are completely rational and risk-neutral, and information in the supply chain is symmetrical, and out of stock is not allowed in the system. In order to differentiate easily, the model uses subscript symbols of $r, l, T$ to represent crossborder e-commerce retailer, overseas warehouse logistics provider and supply chains, and superscript symbols of 1 and 2 to represent the corresponding parameters of Stackelberg and cooperative games respectively.

\section{Modelling formulation}

\subsection{Logistics provider dominated Stackelberg game}

In the Stackelberg game scenario, the third-party overseas warehouse provider is considered as the leader and the cross-border e-commerce retailer as the follower.

The profit of logistics provider is

$$
\pi_{l}=(L-m) \cdot(A-a P+b e)-\frac{k e^{2}}{2}
$$

The total profit of cross-border e-commerce retailer in finite time horizon is

$$
\pi_{r}=(P-w-L) \cdot(A-a P+b e)
$$

Taking the first-order derivative of $\pi_{r}$ of Eq.(1) with respect to $P$, we have

$$
\frac{\partial \pi_{r}}{\partial P}=(A-a P+b e)-a(P-w-L)
$$

Taking the second-order derivative of $\pi_{r}$ with respect to $P$, we have $\frac{\partial^{2} \pi_{r}}{\partial P^{2}}=-2 a<0$. This implies that $\pi_{r}$ is concave in $P$. Solving $\frac{\partial \pi_{r}}{\partial P}=0$, the optimal selling price yields,

$$
P^{1^{*}}=\frac{1}{2 a}(A+b e+a w+a L)
$$

Substituting Eq.(3) into Eq.(1), we have the profit of the logistics provider in the Stackelberg game as

$$
\pi_{l}=\frac{1}{2}(L-m) \cdot(A+b e-a w-a L)-\frac{k e^{2}}{2}
$$

Taking the first partial derivatives of $\pi_{l}$ with respect to $L$ and $e$ yields

$$
\begin{gathered}
\frac{\partial \pi_{l}}{\partial L}=\frac{1}{2}(A+b e-a w+a m-2 a L) \\
\frac{\partial \pi_{l}}{\partial e}=\frac{b}{2}(L-m)-k e
\end{gathered}
$$

Taking the second partial derivatives of $\pi_{l}$ with respect to $\mathrm{L}$ and $\mathrm{S}$, we further have

$$
\begin{aligned}
& \frac{\partial^{2} \pi_{l}}{\partial L^{2}}=-a, \frac{\partial^{2} \pi_{l}}{\partial e^{2}}=-k \text { and } \frac{\partial^{2} \pi_{l}}{\partial L \partial e}=\frac{b}{2} . \\
& \text { Hessian matrix } H_{1}=\left[\begin{array}{rr}
-a & \frac{b}{2} \\
\frac{b}{2} & -k
\end{array}\right]=a k-\frac{1}{4} b^{2}>0,
\end{aligned}
$$

which implies that the profit of the logistics provider is jointly concave in $L$ and $e$.

Solving $\frac{\partial \pi_{l}}{\partial L}=0, \frac{\partial \pi_{l}}{\partial e}=0$, the optimal logistics price and freshness level yields respectively.

$$
\begin{gathered}
L^{1^{*}}=\frac{2 k(A-a w)+\left(2 a k-b^{2}\right) m}{4 a k-b^{2}} \\
e^{1^{*}}=\frac{b(A-a w-a m)}{4 a k-b^{2}}
\end{gathered}
$$

Solving Eq.(3) yields

$$
P^{1^{*}}=\frac{3 a k A+\left(a k-b^{2}\right)(a w+a m)}{a\left(4 a k-b^{2}\right)}
$$

Solving Eqs.(1) and (2) yields

$$
\begin{gathered}
\pi_{l}^{1^{*}}=\frac{k(A-a w-a m)^{2}}{2\left(4 a k-b^{2}\right)} \\
\pi_{r}^{1^{*}}=\frac{a k^{2}(A-a w-a m)^{2}}{\left(4 a k-b^{2}\right)^{2}} \\
\pi_{T}^{1^{*}}=\frac{k\left(6 a k-b^{2}\right)(A-a w-a m)^{2}}{2\left(4 a k-b^{2}\right)^{2}}
\end{gathered}
$$

\subsection{Cooperative game}

In the cooperative game, the cross-border e-commerce retailer and overseas warehouse provider are vertically integrated in the same system. The objective of the supply chain is to determine the optimal selling price, the freshness level to maximize the total profit. The total profit of the supply chain in finite time horizon is

$$
\begin{aligned}
& \pi_{T}=\pi_{r}+\pi_{l}=(P-w-m)(A-a P+b e)-\frac{1}{2} k e^{2} \\
& \frac{\partial \pi_{T}}{\partial P}=(A-2 a P+b e)+a(w+m), \quad \frac{\partial^{2} \pi_{T}}{\partial P^{2}}=-2 a,
\end{aligned}
$$




$$
\begin{gathered}
\frac{\partial \pi_{T}}{\partial e}=b(P-w-m)-k e, \quad \frac{\partial^{2} \pi_{T}}{\partial e^{2}}=-k, \text { and } \frac{\partial^{2} \pi_{T}}{\partial P \partial e}=b . \\
H_{2}=\left[\begin{array}{cc}
-2 a & b \\
b & -k
\end{array}\right]=\mathbf{2} a k-b^{2}>0 \text {, which implies that }
\end{gathered}
$$$$
\pi_{T} \text { is jointly concave in } e \text { and } P \text {. Solving }
$$
$\frac{\partial \pi_{T}}{\partial P}=0, \frac{\partial \pi_{T}}{\partial e}=0$, the optimal selling price and the freshness level can be obtained respectively when the supply chain profit is maximized.

$$
\begin{gathered}
P^{2^{*}}=\frac{k(A+a w+a m)-b^{2}(w+m)}{2 a k-b^{2}} \\
e^{2^{*}}=\frac{b(A-a w-a m)}{2 a k-b^{2}}
\end{gathered}
$$

Solving Eq.(8),we obtain the optimal total profit of the supply chain,

$$
\pi_{T}^{2^{*}}=\frac{k(A-a w-a m)^{2}}{2\left(2 a k-b^{2}\right)}
$$

Applying the two-person cooperative game model with transferable payment, the profits of cross-border ecommerce retailer and logistics providers are respectively as the follows,

$$
\begin{gathered}
\pi_{r}^{2^{*}}=\frac{a k^{2}\left(3 a k-b^{2}\right)(A-a w-a m)^{2}}{\left(2 a k-b^{2}\right)\left(4 a k-b^{2}\right)^{2}} \\
\pi_{l}^{2^{*}}=\frac{k\left(10 a^{2} k^{2}-6 a k b^{2}+b^{4}\right)(A-a w-a m)^{2}}{2\left(2 a k-b^{2}\right)\left(4 a k-b^{2}\right)^{2}}
\end{gathered}
$$

Solving Eq.(1) yields

$$
L^{2^{*}}=\frac{\left(10 a^{2} k^{2}-6 a k b^{2}+b^{4}\right)(A-a w-a m)}{2 a\left(4 a k-b^{2}\right)^{2}}+\frac{b^{2}(A-a w-a m)}{2 a\left(2 a k-b^{2}\right)}+m
$$

\subsection{Comparison of two models}

Comparing the optimal decision variables of cold chain product supply chain between cooperative game and Stackelberg game, it is found that:

Proposition 1 The optimal freshness level in the cooperative game is higher than that in the Stackelberg game. That is, $e^{2^{*}}>e^{1^{*}}$.

Prove:

$$
\because 2 a k-b^{2}<4 a k-b^{2} \therefore \frac{1}{2 a k-b^{2} T}>\frac{1}{4 a k-b^{2} T}
$$

Proposition 2 When $2\left(2 a k-b^{2}\right)^{2}>a k\left(2 a k+b^{2}\right)$ holds, applying the two-person cooperative game model with transferable payment, logistics price of cooperative game is less than the Stackelberg game, i.e. $L^{2^{*}}<L^{1^{*}}$.

Prove:

$$
\begin{aligned}
& L^{2^{*}}-L^{1^{*}}=\frac{\left(10 a^{2} k^{2}-6 a k b^{2}+b^{4}\right)(A-a w-a m)}{2 a\left(4 a k-b^{2}\right)^{2}} \\
& +\frac{b^{2}(A-a w-a m)}{2 a\left(2 a k-b^{2}\right)}+m-\frac{2 k(A-a w)+\left(2 a k-b^{2}\right) m}{4 a k-b^{2}} \\
& =\frac{-k\left[2\left(2 a k-b^{2}\right)^{2}-a k\left(2 a k+b^{2}\right)\right]}{\left(2 a k-b^{2}\right)\left(4 a k-b^{2}\right)^{2}} \cdot(A-a w-a m)
\end{aligned}
$$

As the system assumes no shortage, the total market demand for cross-border e-commerce retailer is more than zero, that is, $A-a P+b e>0$, and hence,

$A+b e>a P, A+b e>a w+a m$.

It is also assumed that when there is no freshness difference in the logistics market, the market demand is still more than zero, that is, the total market demand of cross-border e-commerce retailer degraded to $A-a P>0$, when $b=0$. Thus, $A-a w-a m>0$.

Proposition 2 obtains evidence.

Proposition 3 Both the total optimal profits of the supply chain and the profits of two parties in the cooperative game are higher than those in the Stackelberg game, that is

$$
\pi_{T}^{2^{*}}>\pi_{T}^{1^{*}}, \pi_{r}^{2^{*}}>\pi_{r}^{1^{*}}, \pi_{l}^{2^{*}}>\pi_{l}^{1^{*}}
$$

Prove:

$$
\begin{aligned}
& \begin{aligned}
\pi_{r}^{2^{*}}-\pi_{r}^{1^{*}} & =\frac{\left[a k^{2}\left(3 a k-b^{2}\right)-a k^{2}\left(2 a k-b^{2}\right)\right](A-a w-a m)^{2}}{\left(2 a k-b^{2}\right)\left(4 a k-b^{2}\right)^{2}} \\
& =\frac{a^{2} k^{3}(A-a w-a m)^{2}}{\left(2 a k-b^{2}\right)\left(4 a k-b^{2}\right)^{2}}>0
\end{aligned} \\
& \pi_{l}^{2^{*}}-\pi_{l}^{1^{*}}=\frac{a^{2} k^{3}(A-a w-a m)^{2}}{\left(2 a k-b^{2}\right)\left(4 a k-b^{2}\right)^{2}}>0 \\
& \text { Hence, } \pi_{T}^{2^{*}}>\pi_{T}^{1^{*}} .
\end{aligned}
$$

\section{Numerical example}

In order to to bear out the validity of the theoretical models, the parameters of cold chain cross-border e-commerce are as in Table 1 .

Table 1. the parameters of the example.

\begin{tabular}{|c|c|c|c|c|c|}
\hline$m$ & $A$ & $a$ & $w$ & $b$ & $k$ \\
\hline 4 & 700 & 8 & 50 & 2 & 35 \\
\hline
\end{tabular}

By MATLAB, the optimal results from Stackelberg game and cooperative game are gained, in terms of the logistics service price, freshness level, product price, and the demand within a finite time horizon, and the profits are obtained as in Table 2.

Table 2. The optimal results.

\begin{tabular}{|c|c|c|c|c|c|c|c|}
\hline Optimal results & $\mathbf{L}$ & $\boldsymbol{e}$ & $\mathbf{P}$ & $\mathbf{D}$ & $\pi_{r}$ & $\pi_{l}$ & $\pi_{T}$ \\
\hline Stackelberg game & 20.81 & 0.48 & 79.21 & 67 & 565 & 1126 & 1691 \\
\hline Cooperative game & 14.57 & 0.96 & 70.78 & 135 & 850 & 1411 & 2261 \\
\hline
\end{tabular}


In Table 2, it shows that, under cooperative game, the freshness level of 0.96 is much more than that of 0.48 in the Stackelberg game; and the optimal prices of logistics service and cold chain product, 14.57 and 70.78, are less than those of the Stackelberg game, which are 20.81 and 79.21 respectively. All of them lead to an increase of demand, and the total profit, compared with the Stackelberg game. Thus, from the system perspective, cooperative game outperforms the Stackelberg game. Applying two-person cooperative game with transferable payment, it is found that both of the profits of the supply chain members are more that of the Stackelberg game. That is, win-win performance of the supply chain members are achieved.

\section{Conclusion}

This paper compares the cooperative game and the Stackelberg game of cross-border e-commerce supply chain composed of one e-commerce retailer and one integrated overseas warehouse logistics provider. Theoretical analysis find that if the cross-border ecommerce retailer establishes cooperative relationship with overseas warehouse logistics providers to optimize decision-making with total system profit, it can not only obtain much more system profit, but also get a higher freshness level from the logistics provider, and make a lower retail price. Through two-person cooperative game of transferable payment, both of the two parties can obtain higher profit than the non-cooperative decision-making of Stackelberg game, meanwhile the logistics price is lower than that of the Stackelberg game. Numerical results verify the validity of the theoretical models.

\section{Acknowledgement}

This research was supported by Shandong Social Science Planning Project Research on the governance structure and optimization of fresh overseas warehouse of cross border e-commerce (21CPYJ27) and National Natural Science Foundation of China Coordination and optimization of multi-echelon cold chain inventory system under compensation mechanism (71672166).

\section{References}

1. Sun, G., Qiao, F., Li, Y., 2017. Research on logistics mode of cross border E-commerce in China. Boletín Técnico, 55(10): 490-497.

2. Ramanathan, R., 2010. The moderating roles of risk and efficiency on the relationship between logistics performance and customer loyalty in e-commerce. Transportation Research Part E: Logistics and Transportation Review, 46(06): 950-962.

3. Lu, Q., Liu, N., 2013. Pricing games of mixed conventional and e-commerce distribution channels. Computers \& Industrial Engineering, 64(1): 122-132.

4. Xiao, T., Xu, T., 2013. Coordinating price and service level decisions for a supply chain with deteriorating item under vendor managed inventory. International Journal of Production Economics, 145(2): 743-752.

5. Zhang, J., Wei, Q., Zhang, Q., et al., 2016. Pricing, service and preservation technology investments policy for deteriorating items under common resource constraints. Computers \& Industrial Engineering, 95: $1-9$.

6. Yu, Y., Xiao, T., 2017. Pricing and cold-chain service level decisions in a fresh agri-products supply chain with logistics outsourcing. Computers \& Industrial Engineering, 111: 56-66.

7. Song, Z., He, S., Xu, G., 2018. Decision and coordination of fresh produce three-layer ecommerce supply chain: A New Framework. IEEE Access, 7: 30465-30486.

8. Li, G., Li, L., \& Sun, J., 2019. Pricing and service effort strategy in a dual-channel supply chain with showrooming effect. Transportation Research Part Elogistics and Transportation Review. 126, 32-48.

9. Choi, T., Li, Y., Xu, L., 2013. Channel leadership, performance and coordination in closed loop supply chains. International Journal of Production Economics, 146(1): 371-380.

10. Jørgensen, S., Zaccour, G., 2014. A survey of gametheoretic models of cooperative advertising. European Journal of Operational Research, , 237(1): $1-14$. 